\title{
Non-reflective inlet conditions for the calculation of unsteady turbulent compressible flows at low Mach number
}

\author{
Yann Moguen ${ }^{1, a}$, Pascal Bruel ${ }^{2}$, Vincent Perrier ${ }^{3}$ And Erik Dick ${ }^{4}$ \\ 1 Université de Pau et des Pays de l'Adour, LMAP and Inria, Cagire Team, IPRA, Avenue de l'Université, 64013 Pau, France \\ 2 CNRS, Université de Pau et des Pays de l'Adour, LMAP and Inria, Cagire Team, IPRA, Avenue de l'Université, 64013 Pau, \\ France \\ 3 Inria, Cagire Team and Université de Pau et des Pays de l'Adour, LMAP, IPRA, Avenue de l'Université, 64013 Pau, France \\ ${ }^{4}$ Ghent University - Department of Flow, Heat and Combustion Mechanics, Sint-Pietersnieuwstraat, 9000 Gent, Belgique
}

Received 20 June 2013, Accepted 31 March 2014

\begin{abstract}
For the unsteady simulation of compressible subsonic flows (Large Eddy Simulation or Direct Numerical Simulation), the proper handling of the inlet boundary is a challenging task. Indeed, inflow generation through imposition of the velocity may lead to a non-physical reflection of the upstream acoustic waves. In the present contribution, a method that allows both filtering of these waves and proper imposition of the required variables is proposed. This method is based on identification of the roles of the temporal rate of change of wave amplitudes at the inlet in the low Mach number regime. The formulation obtained is tested numerically on unsteady one-dimensional flows at low Mach number for which the unsteady inlet velocity signal is purely harmonic or harmonic with the superimposition of synthetic turbulence.
\end{abstract}

Key words: Non-reflective boundary conditions / inlet conditions / low Mach number / turbulence / acoustics

Résumé - Conditions d'entrée non réfléchissantes pour le calcul d'écoulements turbulents compressibles à bas nombre de Mach. Pour le calcul instationnaire d'écoulements turbulents compressibles subsoniques (simulations des grandes échelles ou simulation numérique directe), la prescription de conditions d'entrée satisfaisantes pose problème. En effet, générer l'écoulement en entrée via l'imposition du champ de vitesse peut conduire à une réflexion non physique des ondes acoustiques qui remontent l'écoulement. Dans la présente contribution, une méthode permettant à la fois de filtrer ces ondes et d'imposer les variables requises en entrée est proposée. Cette méthode repose sur l'identification des rôles respectifs des variations d'amplitude des ondes acoustiques et convectives en entrée, à bas nombre de Mach. Elle est testée numériquement pour la simulation d'un écoulement instationnaire à bas nombre de Mach avec un signal d'entrée de type harmonique seul ou combiné avec un signal synthétique de turbulence.

Mots clés : Conditions aux limites non réfléchissantes / conditions d'entrée / bas nombre de Mach / turbulence / acoustique

\section{Introduction}

With compressible subsonic flows, difficulties may arise when prescribing the boundary conditions. Indeed, the imposition of variables such as velocity or pressure may lead to a non-physical reflection of the outgoing acoustic waves at the boundary of the computational domain. The problem is particularly severe for inlet

\footnotetext{
${ }^{a}$ Corresponding author: yann.moguen@univ-pau.fr
}

conditions when the inflow is turbulent, since the turbulence generation is carried out through imposition of time varying inlet variables.

To address this issue, characteristic-based boundary conditions using the temporal rate of change of the wave amplitudes (or, in short, the wave amplitudes) were suggested by Thompson [1] for Euler equations, and Poinsot and Lele [2] for Navier-Stokes equations. According to the latter reference, viscous and transverse terms 
of the Navier-Stokes equations may be disregarded for estimation of the wave amplitudes. This approach, called Locally One-Dimensional Inviscid (LODI), is followed in the present study, where viscous terms are present in the equations solved in the interior of the computational domain. As a special case of the LODI approach, linear relaxation methods, based on Rudy and Strikwerda [3], represent a trade-off between the imposition of variables and the partial reflection of acoustic waves. The linear relaxation applied to the velocity at the inlet results in a low-pass filter for the reflected waves [4]. This allows to circumvent the drift of boundary values that may occur if rough non-reflective boundary conditions are applied [2]. However, since the cut-off frequency is proportional to the relaxation coefficient ${ }^{1}$, velocity imposition and nonreflection at the inlet are incompatible if a basic linear relaxation approach is used.

The relaxation approach was re-examined by Pirozzoli and Colonius [6]. In their study, the relaxation is applied to the characteristic variables corresponding to the ingoing waves, instead of application to the primitive variables, which is the common practice. It was demonstrated both theoretically and numerically that the resulting level of reflection at the boundary is satisfactory. In practice however, some information needed from the outside of the domain may be not available (e.g. pressure at the inlet), which makes the proper implementation of the proposed method problem-dependent.

As a cure to the problem of undesirable reflection, Polifke et al. [7] proposed to detect and eliminate the outgoing acoustic fluctuations. At a flow inlet, the term that carries out that filtering is introduced in the temporal rate of change of the ingoing wave amplitude, written under a linear relaxation form involving the velocity variable. However, (1) the detection of the outgoing acoustic waves requires an additional computational cost, (2) the discrimination between the outgoing acoustic fluctuations and the turbulent fluctuations is tractable only for plane acoustic waves, and (3) the relaxation coefficient must be adjusted for each flow problem considered.

Following considerations of Prosser [8], Guézennec and Poinsot [9] proposed inflow conditions based on the interpretation of low Mach number asymptotic analysis of the acoustic wave amplitudes, under the frozen turbulent inflow hypothesis $[10]^{2}$. An advantage of this approach is to avoid the linear relaxation and the related issue of coefficient tuning, while no drift of the boundary values has been reported. Another advantage is that no detection of the upstream acoustic waves to be filtered is needed. The VFCBC method proposed in [9] is a special case of the LODI approach, that is devoted to the injection of inertial disturbances at the inlet. The inadequacy of this method for acoustic waves injection was noticed in [9]. If acoustic waves only have to be generated at the inlet, another

1 This follows immediately from the calculation of the reflection coefficient at the inlet; see $[5,6]$ for this calculation.

${ }^{2}$ We refer here to the method called Vortical-Flow Characteristic Boundary Condition (VFCBC) in [9]. approach was recommended in this study ${ }^{3}$. The inadequacy of this other approach for inertial disturbances injection was evidenced by numerical experiments. To use the VFCBC method proposed in [9] for inertial disturbances injection, it is thus necessary to consider a priori that the signal to be generated is of purely inertial nature. However, this may be unclear in practice, for example if turbulent fluctuations are superimposed on an acoustic signal. It will be shown here that the VFCBC formulation leads in that case to a non-satisfactory estimation of the acoustic energy level.

The notion of frozen turbulent inflow refers to the idea of convecting inertial disturbances with the mean flow velocity. At low Mach number, the convective and acoustic scales are very different. In that case, since turbulent fluctuations are considered as inertial disturbances, they must be injected at the convective scale only. This idea is central in the approaches developed by Prosser [8] and Guézennec and Poinsot [9], and will be adopted in the present study.

The boundary handling at an inlet flow proposed in the present study preserves the advantages of the VFCBC method of [9] for inertial disturbances injection, while avoiding assumptions on either the inertial or the acoustic nature of the signal to be generated. Moreover, it allows for the proper calculation of the acoustic field in the computational domain when the injected signal is of purely acoustic nature, or when turbulent fluctuations are superimposed on an acoustic signal. The method has common features with the NR-NSCBC method proposed in [9] for acoustic waves injection. Both methods are based on the following simple physical principle: at the inlet, the gap observed (if any) between the target velocity value and its current value is attributed solely to the upstream waves, which are necessarily acoustic. We will show that the entering acoustic wave amplitude thus obtained conforms to the multiple scales low Mach number asymptotics, independently of the frozen turbulent inflow hypothesis. By contrast, the entropic wave amplitude proposed at the inlet is derived both from the low Mach number asymptotics and the frozen turbulent inflow hypothesis. The resulting set of wave amplitudes to be prescribed differs from that of the NR-NSCBC and VFCBC methods. It is an original formulation of the inlet conditions that is expected to avoid the above mentioned drawbacks of these methods.

The numerical experiments presented here concern low Mach number flows. The numerical method employed relies on a pressure correction approach, with a SIMPLE-type pressure-velocity coupling combined with a momentum interpolation method (see [11] for a detailed description). Small time-steps are used to allow for the proper handling of the inflow turbulent fluctuations when these are present. The often mentioned problems of momentum interpolation methods when a small time-step is used for unsteady calculations (see e.g. [12]) are avoided thanks to the transporting velocity proposed in [11].

\footnotetext{
3 We refer here to the method called Non-Reflecting NavierStokes Characteristic Boundary Condition (NR-NSCBC) in [9].
} 


\section{Formulation of the inlet conditions}

Consider a one-dimensional flow of air. The wave amplitudes are given by

$$
\begin{aligned}
\mathcal{L}_{1} & =(v-c)\left(\frac{1}{\varrho c} \partial_{x} p-\partial_{x} v\right) \\
\mathcal{L}_{2} & =v\left(\partial_{x} \varrho-\frac{1}{c^{2}} \partial_{x} p\right) \\
\mathcal{L}_{3} & =(v+c)\left(\frac{1}{\varrho c} \partial_{x} p+\partial_{x} v\right)
\end{aligned}
$$

$\mathcal{L}_{1}$ and $\mathcal{L}_{3}$ are the upstream and downstream acoustic wave amplitudes, respectively, and $\mathcal{L}_{2}$ is the entropic wave amplitude [2]. These wave amplitudes satisfy the LODI equations, namely

$$
\begin{aligned}
\partial_{t} \varrho+\frac{\varrho}{2 c}\left(\mathcal{L}_{1}+\mathcal{L}_{3}\right)+\mathcal{L}_{2} & =0 \\
\partial_{t} v+\frac{1}{2}\left(\mathcal{L}_{3}-\mathcal{L}_{1}\right) & =0 \\
\partial_{t} p+\frac{\varrho c}{2}\left(\mathcal{L}_{1}+\mathcal{L}_{3}\right) & =0
\end{aligned}
$$

The first principle adopted for the inlet conditions is to consider that the possible gap between the target velocity $v^{\dagger}$ and the current value of the velocity variable $v$ is due to the upstream acoustic waves only, which correspond to $\mathcal{L}_{1}$. This may be implemented by setting

$$
\mathcal{L}_{3}=-2 \partial_{t} v^{\dagger}
$$

in equation $(2 \mathrm{~b})$, which then implies

$$
\partial_{t}\left(v-v^{\dagger}\right)+\frac{1}{2}\left(0-\mathcal{L}_{1}\right)=0
$$

Since $\hat{\mathcal{L}_{3}}(\omega)=2 \mathrm{i} \omega \hat{v^{\dagger}}(\omega)$, where $\hat{r}$ designates the Fourier transform in time, the inlet reflection coefficient $\hat{\mathcal{L}}_{3} / \hat{\mathcal{L}}_{1}$ is zero $^{4}$. It is worth noticing that this result holds independently of the frequency of the outgoing acoustic signal. Thus, from equation (3), the suitable non-reflective properties at the inlet are immediately satisfied.

An equation similar to equation (3) was suggested but not used by Polifke et al. [7] in presence of external acoustic signals. To obtain a non-reflective inlet treatment that allows to generate vortices and turbulence at the inflow, Guézennec and Poinsot [9] proposed to adopt the equations

$$
\mathcal{L}_{3}=-\partial_{t} v^{\dagger} \quad \text { and } \quad \mathcal{L}_{2}=0
$$

This boundary condition, called Vortical-Flow Characteristic Boundary Condition (VFCBC), arises from an interpretation of the low Mach number asymptotic multiple scales expansions of the $\mathcal{L}_{i}$ 's, with Taylor's frozen turbulence hypothesis [10] assumed for the inflow. Namely,

\footnotetext{
4 To define the inlet reflection coefficient, the target velocity $v^{\dagger}$ is considered as constant, since the acoustic waves generated by the fluctuations of $v^{\dagger}$ are not reflected waves.
}

it is established by Prosser [8] that under this assumption $\partial_{t} p=0$ and $\partial_{t} \varrho=0$ at the leading order of the low Mach number expansions. It is deduced in [9] that $\mathcal{L}_{3}=$ $-\left[\partial_{t} p /(\varrho c)+\partial_{t} v^{\dagger}\right]=-\partial_{t} v^{\dagger}$ and $\mathcal{L}_{2}=\partial_{t} p / c^{2}-\partial_{t} \varrho=0$. Note however that, at the leading order, $\partial_{t} p=\mathrm{d}_{t} p^{(0)}$, where $p^{(0)}$ is the thermodynamic part of the pressure ${ }^{5}$. Therefore, setting $\partial_{t} p=0$ for the total pressure $p$ implies that the acoustic and the hydrodynamic parts of the pressure $^{5}$ are also constant in time, which is a restrictive simplification. To make clear this latter point, the reader is referred to Appendix A.

The expression of $\mathcal{L}_{3}$ in equation (5) and our proposed expression in (3) differ by the factor 2 . The non-reflective property of the inlet is thus satisfied with the VFCBC method as with equation (3), since the same argument applies. However, the difference by the factor 2 does obviously affect the generated signal, and in particular its acoustic features since $\mathcal{L}_{3}$ is the entering acoustic waves amplitude. This point, already mentioned in [9], will be numerically illustrated in the following.

A method called Non-Reflecting Navier-Stokes Characteristic Boundary Condition (NR-NSCBC) was also studied in [9]. The equations for NR-NSCBC are

$$
\mathcal{L}_{3}=-2 \partial_{t} v^{\dagger} \quad \text { and } \quad \mathcal{L}_{2}=-(\gamma-1) \frac{\varrho}{c} \partial_{t} v^{\dagger}
$$

In equations (6), the expression of $\mathcal{L}_{2}$ is derived from the LODI equation of temperature. It is assumed that $\partial_{t} T^{\dagger}=0$, where $T^{\dagger}$ is the target value of temperature, and that the gap between the target and current values of temperature is due to the upstream acoustic waves only. In [9], numerical experiments indicate that this approach is inadequate for the introduction of vortices or turbulence. This inadequacy is confirmed by the multiple scales low Mach number analysis given in Appendix A. Indeed, under the frozen turbulent inflow hypothesis expressed in equations (A.18), the entropic wave amplitude is zero up to the zeroth-order terms (see Eqs. (A.21)). Setting $\mathcal{L}_{2}=0$ is thus justified for turbulence injection at low Mach number, which conforms to the analysis by Guézennec and Poinsot [9] and the numerical results shown herein. Another enlightenment arising from the asymptotic approach concerns the entering acoustic wave amplitude $\mathcal{L}_{3}$ (see Eqs. (A.25)). Injecting asymptotic expansions in equation (4), equation (3) is retrieved at the leading order at the acoustic scale for the zeroth-order target velocity (see Eq. (A.25b)). The target velocity fluctuations are thus imposed primarily at the acoustic scale. If the numerical method is able to capture correctly these fluctuations, one can expect that the acoustic waves produced by the velocity fluctuations at the inlet are properly taken into account. Note also that the asymptotic properties of $\mathcal{L}_{3}$ given by equations (A.25) are derived without the frozen turbulent inflow hypothesis (Eqs. (A.18)), which is only used for the derivation of $\mathcal{L}_{2}$.

The three non-reflective methods that we consider for inlet conditions are recapitulated in Table 1. The method

\footnotetext{
${ }^{5}$ See e.g. [8] or Appendix A.
} 


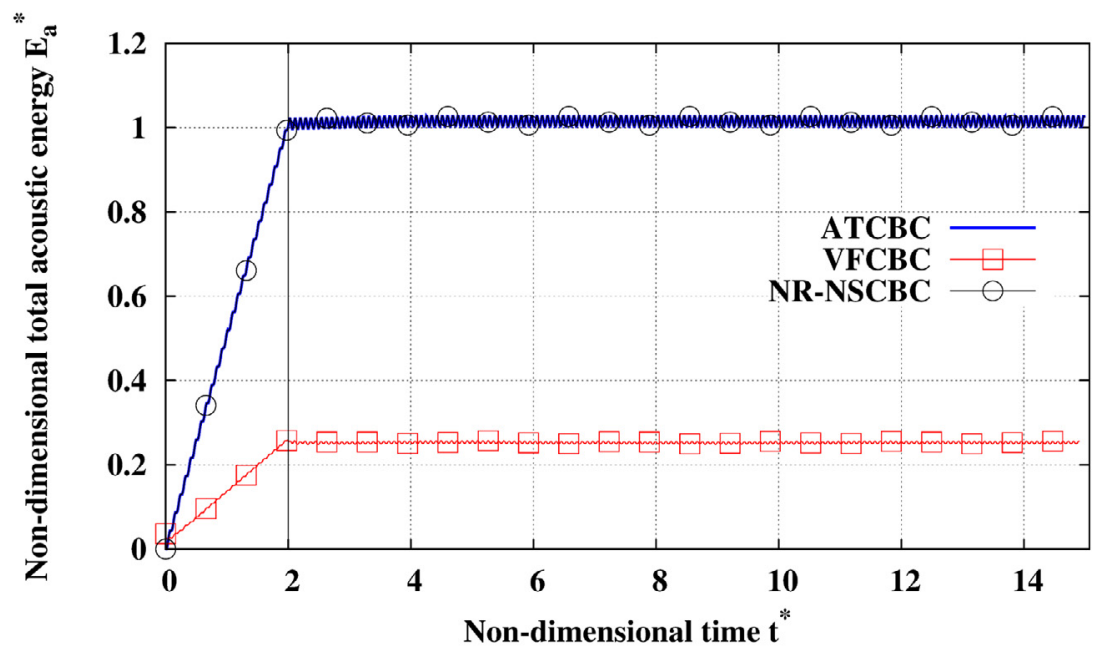

Fig. 1. Test of Section 3: Harmonic inlet velocity. Non-dimensional time evolution of the non-dimensional total acoustic energy $E_{a}^{*}$. Results by ATCBC and NR-NSCBC almost coincide.

Table 1. Ingoing wave amplitudes at the inlet for the three methods considered in 1-D.

\begin{tabular}{cccc}
\hline Method & ATCBC & VFCBC & NR-NSCBC \\
\hline $\mathcal{L}_{\mathbf{2}}$ & 0 & 0 & $-(\gamma-1) \frac{\rho}{c} \partial_{t} v^{\dagger}$ \\
$\mathcal{L}_{\mathbf{3}}$ & $-2 \partial_{t} v^{\dagger}$ & $-\partial_{t} v^{\dagger}$ & $-2 \partial_{t} v^{\dagger}$ \\
\hline
\end{tabular}

Table 2. Ingoing wave amplitudes at the inlet for the ATCBC method in 3-D.

\begin{tabular}{ccccc}
\hline Method & $\mathcal{L}_{2}$ & $\mathcal{L}_{3}$ & $\mathcal{L}_{4}$ & $\mathcal{L}_{5}$ \\
\hline ATCBC & 0 & $-\partial_{t} v^{\dagger}$ & $-\partial_{t} w^{\dagger}$ & $-2 \partial_{t} u^{\dagger}$ \\
\hline
\end{tabular}

proposed here is called ATCBC for Acoustic Turbulence Characteristic Boundary Conditions.

To conclude this section, let us give the formulation of the ATCBC method in the three-dimensional case. Suppose that the inlet plane is $(y, z)$. The velocity components are designated by $(u, v, w)$ and the symbol $\dagger$ denotes the target values. The wave amplitudes for vorticity are obtained by taking $v=v^{\dagger}$ and $w=w^{\dagger}$ in the LODI equations: $\partial_{t} v+\mathcal{L}_{3}=0$ and $\partial_{t} w+\mathcal{L}_{4}=0$. Indeed, since $\mathcal{L}_{1}$ is not involved in the resulting equations, these are nonreflective, as already noted in [9]. The 3-D formulation of the ATCBC method thus obtained is given in Table 2.

\section{Test with harmonic inlet velocity}

As a first test, we consider a flow of air with the specific heats ratio $\gamma=1.4$, in a pipe of length $L=100 \mathrm{~m}$ divided into 5000 cells of equal length. The inlet velocity is harmonic, $v^{\dagger}(t)=\langle v\rangle[1+A \sin (2 \pi f t)]$, with $A=10^{-2}, f=20 \mathrm{~Hz}$ and $\langle v\rangle=0.30886 \mathrm{~m} . \mathrm{s}^{-1}$. Initially, there is no flow in the pipe, with $p=101300 \mathrm{~Pa}$ and $\varrho=1.2 \mathrm{~kg} \cdot \mathrm{m}^{-3}$. At the outlet, we write $\mathcal{L}_{1}=K_{p}\left(p-p^{\dagger}\right)$ with $p^{\dagger}=101300 \mathrm{~Pa}$. The acoustic waves are reflected at the outlet. Here, in practice, this is achieved by taking $K_{p}=10^{3}$. The Mach number of the mean flow is approximately $10^{-3}$.
Values of amplitude $A$ and frequency $f$ are such that an exact solution can be derived from linear acoustics. Before the oscillating signal has reached the outlet, this exact solution is thus obtained by its advection at the speed $v_{0}+c_{0}$, with $c_{0}=\sqrt{\gamma p_{0} / \varrho_{0}}$, where $\varrho_{0}, v_{0}$ and $p_{0}$ designate the density, the velocity and the pressure of the mean flow. After the signal has reached the outlet, the exact solution is obtained by the linear superposition of the downstream propagating signal and the upstream propagating signal which is reflected.

The non-reflective inlet property is checked by calculating the total acoustic energy in the pipe,

$$
E_{a}=\frac{1}{2} \int_{0}^{L}\left\{\varrho_{0}(\delta v)^{2}+\frac{(\delta p)^{2}}{\varrho_{0} c_{0}^{2}}\right\}
$$

where $\delta p=p-p_{0}$ and $\delta v=v-v_{0}$.

To allow for the assessment of the applicability and significance of the numerical results, non-dimensional quantities $x^{*}, t^{*}, v^{*}, E_{a}^{*}$ are considered for length, duration, velocity and total acoustic energy, respectively. To define the non-dimensional length $x^{*}$, the reference length is defined as the wavelength of the acoustic wave generated at the left side of the computational domain. The reference duration is defined as the time needed for an acoustic wave to travel over the computational domain. The non-dimensional velocity $v^{*}$ is defined with $v_{0}$ as reference velocity. The reference total acoustic energy is the total acoustic energy at the time $t^{*}=2$ necessary for forward and backward travel of the acoustic wave front.

In Figure 1, the simulation length is about 15 times the duration necessary for an acoustic wave to cross the pipe, and the full vertical line indicates $t^{*}=2$. We observe that all methods lead to a non-reflective inlet. The acoustic energy level reached in the steady phase is significantly higher with equation (3) than with the VFCBC method, however. The reason of the difference is evidenced in Figure 2, where the inlet is at the left side of the computational domain. The amplitude of the imposed velocity 




Fig. 2. Test of Section 3: Harmonic inlet velocity. Non-dimensional velocity field at non-dimensional time $t^{*}=0.7$. Results by ATCBC and NR-NSCBC coincide.



Fig. 3. Test of Section 3: Harmonic inlet velocity. Non-dimensional velocity field at non-dimensional time $t^{*}=2.5$. Results by ATCBC and NR-NSCBC coincide.

is correct only when equation (3) is used. The amplitude obtained with the VFCBC method is half of the correct one. This conforms with the following basic analysis of equations (2). At $t^{*}=0.7$, no acoustic wave has been yet reflected at the outlet, so that $\mathcal{L}_{1}=0$. In this case, from equation $(2 \mathrm{~b}), \mathcal{L}_{3}=-2 \partial_{t} v$. Provided that $v=v^{\dagger}$ at $t=0$, one has $v=v^{\dagger}$ if $\mathcal{L}_{3}=-2 \partial_{t} v^{\dagger}$ (see Eq. (3)). Similarly, $v=v^{\dagger} / 2$ if $\mathcal{L}_{3}=-\partial_{t} v^{\dagger}$ (see Eqs. (5)). The inadequacy for acoustic waves injection of the VFCBC method, already mentioned in reference [9], is thus evidenced. In contrast, the ATCBC and NR-NSCBC methods lead to satisfactory results.

In Figure 3, the velocity field is displayed at $t^{*}=2.5$. The acoustic wave reflected at the outlet has then reached the inlet again. The amplitude of the calculated velocity is twice the amplitude of the exact incident wave, also displayed in Figure 3. This again conforms with a basic analysis of equations $(2)$, since at $t^{*}=2.5, \mathcal{L}_{1}=-\mathcal{L}_{3}$ if the outlet is fully reflective and if no reflection of left-going acoustic wave occurs at the inlet. Then, $\mathcal{L}_{3}=-\partial_{t} v$ from equation (2b). With equation (3), one obtains $v=2 v^{\dagger}$ under suitable initial conditions, which is observed in
Figure $3^{6}$. The absence of reflection for acoustic waves at the inlet with the ATCBC and NR-NSCBC methods is thus illustrated.

\section{Turbulent velocity generation}

We follow here Biferale et al. [13], where a method based on the solution of Langevin stochastic differential equations is proposed. The inlet velocity is written as $v^{\dagger}(t)=\langle v\rangle+v^{\prime}(t)$, with

$$
v^{\prime}(t)=\sum_{k=1}^{K} g_{k}(t) \chi_{1}(t) \chi_{2}(t) \ldots \chi_{k}(t)
$$

In equation (8), each $g_{k}(t)$ is solution of the linear Langevin equation

$$
\mathrm{d} X_{t}=-\frac{1}{\Lambda_{k}} X_{t} \mathrm{~d} t+\sigma_{k} \sqrt{\frac{2}{\Lambda_{k}}} \mathrm{~d} W_{t}
$$

${ }^{6}$ Note however that for values of $t^{*}$ larger than 2.5 , the amplitude of the velocity field may be not twice the one of the incident wave (not shown). This is due to the phase shift at the reflective outlet; see [5]. 


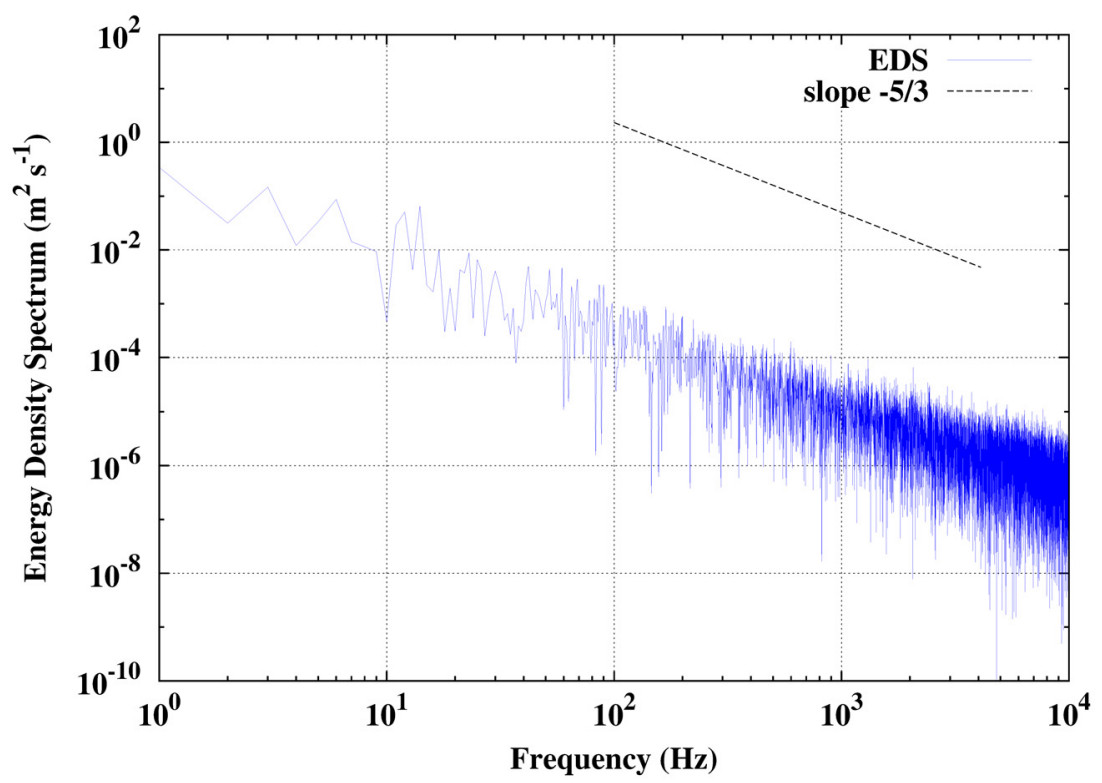

Fig. 4. Energy density spectrum of $v^{\prime}$, cf. equation (8). $K=15, b=0.9,\langle v\rangle=0.30886 \mathrm{~m} \cdot \mathrm{s}^{-1}$. Simulation duration: $1 \mathrm{~s}$.

and each $\chi_{j}(t)$ is solution of the non-linear Langevin equation

$$
\mathrm{d} X_{t}=\frac{2}{\Lambda_{j}} \frac{\mathrm{d} V}{\mathrm{~d} X_{t}} \mathrm{~d} t+\sqrt{\frac{2}{\Lambda_{j}}} \mathrm{~d} W_{t}
$$

$W_{t}$ designates the Wiener process and we set $\Lambda_{k}=2^{-k}$ and $\sigma_{k}=\left(\langle v\rangle \Lambda_{k}\right)^{1 / 3}$ for $k=1,2, \ldots, K$. Further, setting $0<b<1$ :

$$
V(x)= \begin{cases}-2 \ln x & \text { if }(1-b)^{1 / 3}<x<(1+b)^{1 / 3} \\ \infty & \text { else }\end{cases}
$$

The structure functions of the generated field, written in time through the Taylor hypothesis, $S_{q}(\tau)=\langle| \delta v(t+\tau)-$ $\left.\left.\delta v(t)\right|^{q}\right\rangle$, are of order $\tau^{\zeta_{q}}$ with $\zeta_{q}=h q-\log _{2}\left\langle x^{q}\right\rangle$, which characterises the suitable multiaffine structure of the signal (see [13]). In particular, the slope of the energy density spectrum of the generated $v^{\prime}$ matches the theoretical slope of $-5 / 3$ (see Fig. 4 ).

\section{Test with harmonic inlet velocity and superimposed turbulence}

In this section, the aim is to illustrate on a toy problem how to impose a turbulent velocity signal at the inlet of the computational domain, while maintaining the nonreflective property already checked in Section 3 for a harmonic inlet velocity. The computational domain is the one of Section 3, with the same initial and outlet conditions. At the inlet, the turbulent velocity terms are generated with the method of Section 4 and introduced as perturbations of the target velocity adopted in Section 3, as

$$
v^{\dagger}(t)=\langle v\rangle[1+A \sin (2 \pi f t)]+v^{\prime}(t)
$$

The values of $\langle v\rangle, A$ and $f$ are the same as in Section 3 . Equations (9) and (10) are solved with $K=15$ octaves and $b=0.9$. Practically, the time-step $\Delta t$ must be about $2^{-K} / 10$ (see [14]), so that the value of the convective CFL number is $\mathrm{CFL}_{v}=v \Delta t / \Delta x \simeq 0.30886 \times 3 \times 10^{-6} /(2 \times$ $\left.10^{-2}\right) \simeq 5 \times 10^{-5}$, and the acoustic CFL number is $\mathrm{CFL}_{v+c}=\left(1+\mathrm{M}^{-1}\right) \mathrm{CFL}_{v} \simeq 5 \times 10^{-2}$. For such small time-steps, the convenience of the numerical method used in the present paper relies on the time consistency property of the transporting velocity, as evidenced in [11].

Results are displayed with the non-dimensionalisation defined in Section 3. The velocity fields obtained by the ATCBC and the VFCBC methods are shown in Figure 5 at time $t^{*}=2.5$. For both methods, no reflection is visible at the inlet. The non-reflective behaviour of the inlet is confirmed by the total acoustic energy evolution shown in Figure 7. Note that results by the NR-NSCBC method coincide with those by the ATCBC method for the present case, where the turbulence intensity is about $1 \%$ (see Figs. 6 and 7).

If the turbulence intensity of the inertial fluctuations superimposed on an acoustic signal is sufficiently small, it is reasonable to expect that the resulting total acoustic energy level is close to the one obtained without fluctuations. In Figure 7, the satisfying level of total acoustic energy is obtained only with the ATCBC and the NR-NSCBC methods.

According to the low Mach number multiple scales asymptotic analysis presented in Appendix A, the leading order $\mathcal{L}_{2}$ expression for the NR-NSCBC method involves time variations at the acoustic scale (see Eq. (A.22a)). It is then expected that with the NR-NSCBC method, in the low Mach number regime, acoustic waves are generated through the entropic wave amplitude, in addition to those generated through the acoustic wave amplitude $\mathcal{L}_{3}$. Comparing equations (A.21a)-(A.21b) with equations (A.22a)-(A.22b), the ATCBC method must be practically less "noisy" than the NR-NSCBC method at low Mach number. This is illustrated in Figure 8, where the velocity field is displayed at time $t^{*}=0.7$. 


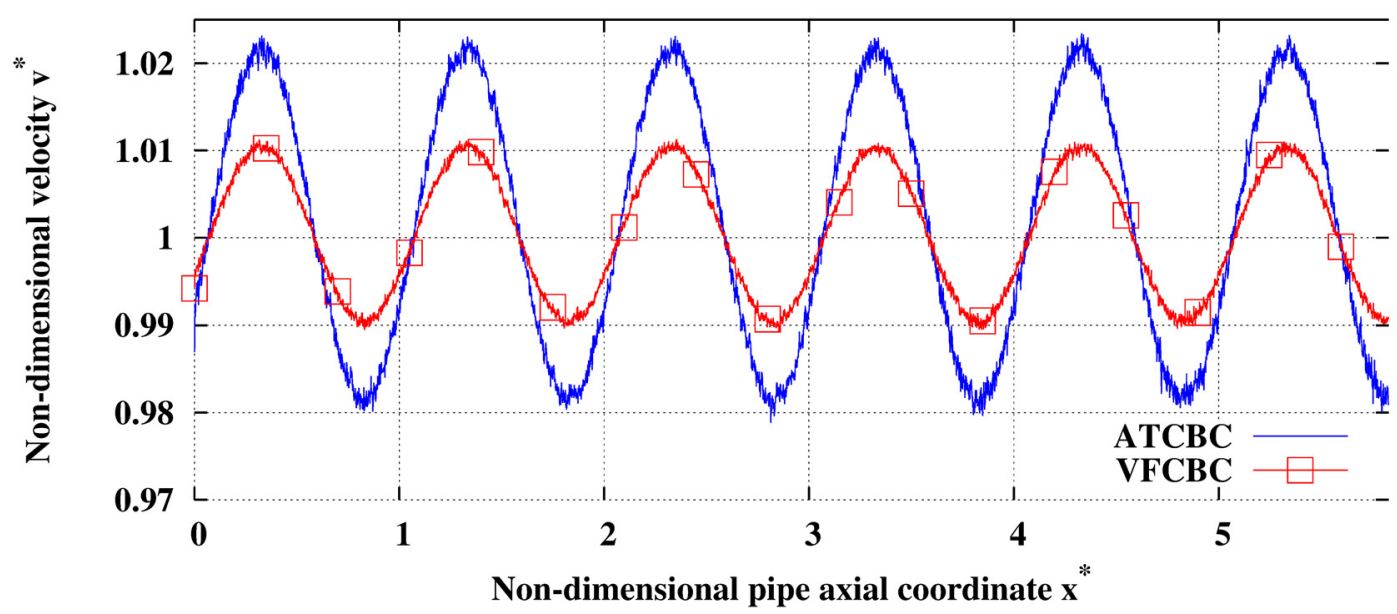

Fig. 5. Test of Section 5: Harmonic inlet velocity and superimposed turbulence. Non-dimensional velocity field at time $t^{*}=2.5$. Turbulence intensity of the entering velocity signal: $1 \%$.

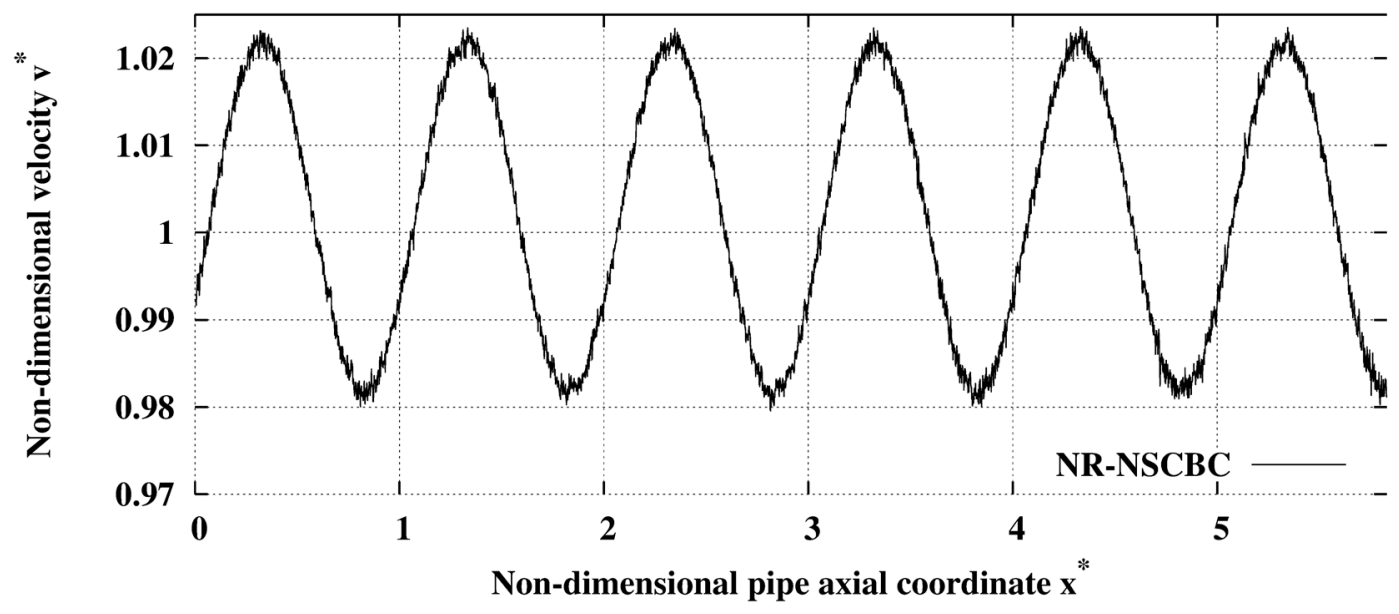

Fig. 6. Test of Section 5: Harmonic inlet velocity and superimposed turbulence. Non-dimensional velocity field at time $t^{*}=2.5$. Same computational parameters as in Figure 5.

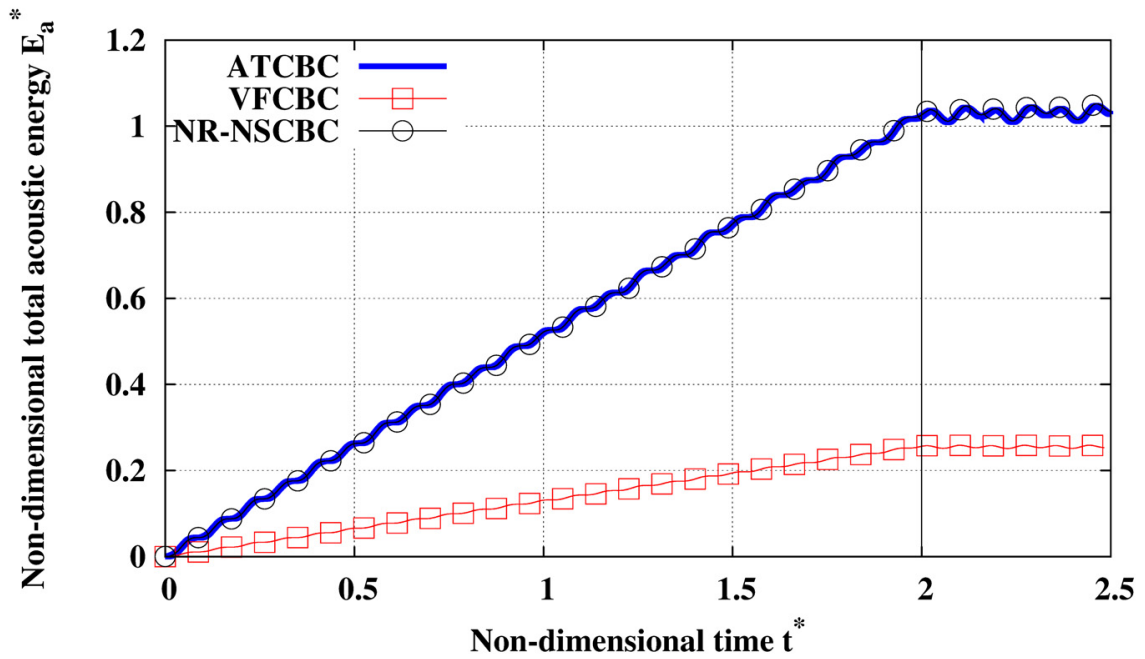

Fig. 7. Test of Section 5: Harmonic inlet velocity and superimposed turbulence. Non-dimensional time evolution of the nondimensional total acoustic energy $E_{a}^{*}$. Results by ATCBC and NR-NSCBC coincide. 


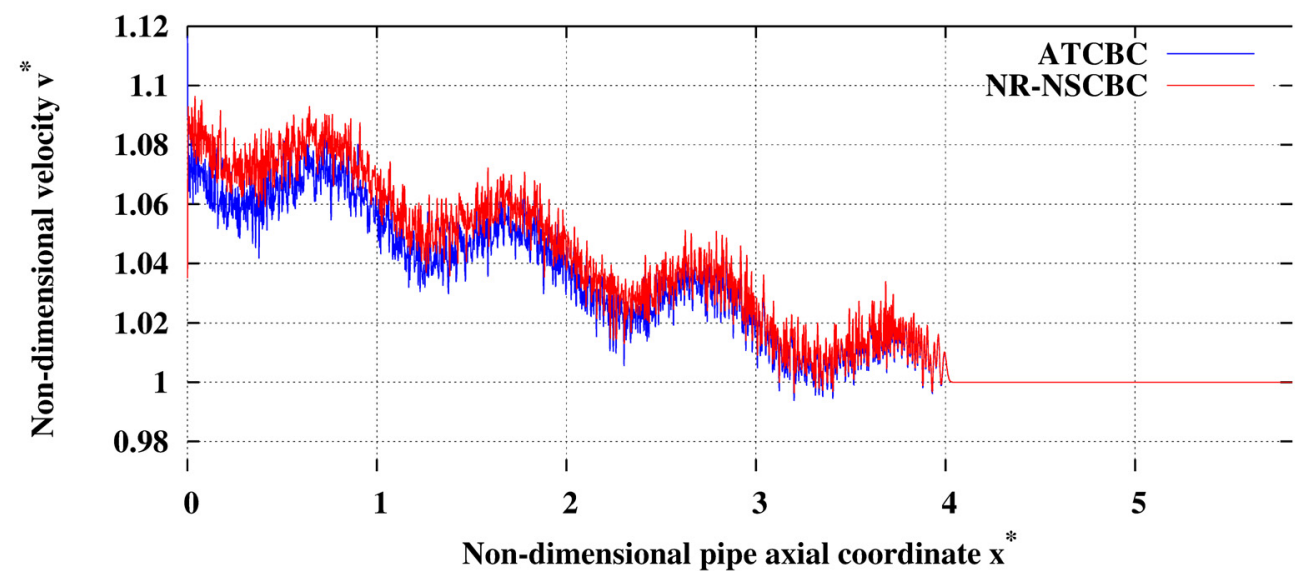

Fig. 8. Test of Section 5: Harmonic inlet velocity and superimposed turbulence. Non-dimensional velocity field at nondimensional time $t^{*}=0.7$. Turbulence intensity of the entering velocity signal: $10 \%$.

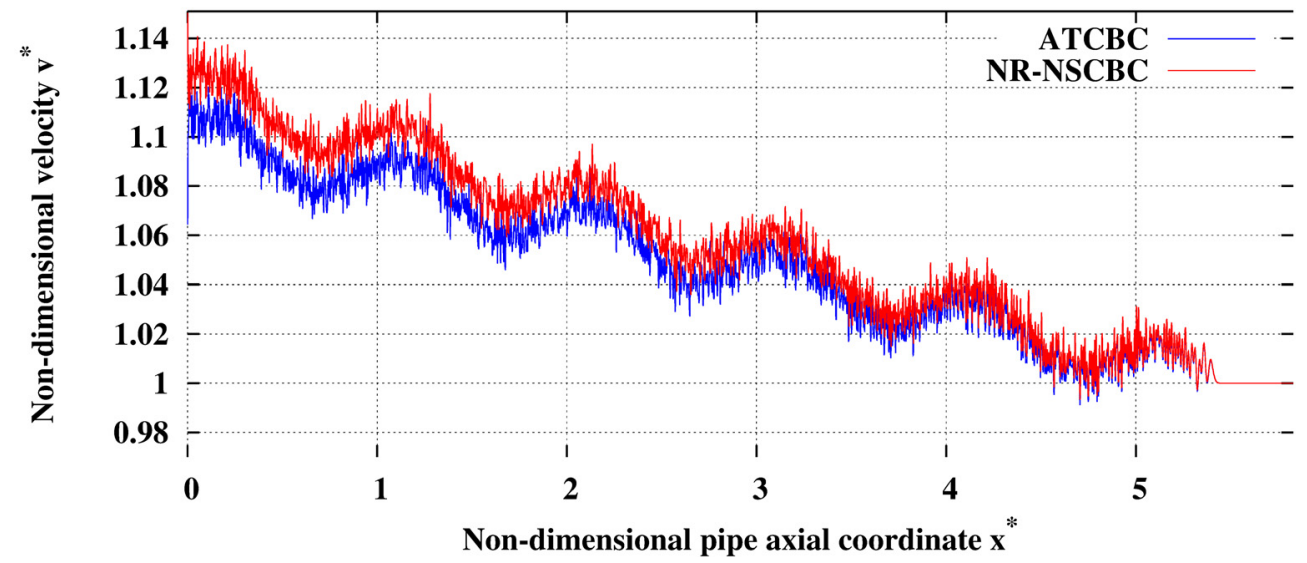

Fig. 9. Test of Section 5: Harmonic inlet velocity and superimposed turbulence. Non-dimensional velocity field at nondimensional time $t^{*}=0.95$. Turbulence intensity of the entering velocity signal: $10 \%$.

For both the ATCBC and the NR-NSCBC methods, the gap between the target and current velocity values at the inlet is due to the upstream acoustic waves generated by the turbulent fluctuations in the interior of the domain (these waves are absent in the harmonic case shown in Fig. 2). The downstream acoustic waves generated by the NR-NSCBC method increase this gap more than with the ATCBC method. At time $t^{*}=0.95$, shown in Figure 9, the discrepancy between results by the ATCBC and the NR-NSCBC methods becomes more visible since the amount of upstream acoustic waves that originate from the turbulent fluctuations is increased. As far as the "noisy" features of the NR-NSCBC method are concerned, these results conform to the analysis by Guézennec and Poinsot [9].

\section{Conclusion}

The objective of the present paper was to demonstrate the importance of the proper identification of the role of the wave amplitudes to obtain non-reflective boundaries in compressible low Mach number flow simulations.
This identification was carried out on the basis of multiple scales low Mach number asymptotics. With the LODI equations solved at the inlet, a simple and effective way to impose a time varying velocity while satisfying nonreflective properties of the inlet, was proposed and tested on simple problems. It was shown that the inclusion of turbulent velocity fluctuations in the velocity imposed at the inlet does not deteriorate the non-reflective behaviour of the inlet. It was also shown that the correct acoustic energy level is obtained by the proposed method for acoustic waves injection. Moreover, when turbulent fluctuations are superimposed on acoustic waves, the correct acoustic energy level was retrieved if the turbulence intensity was sufficiently small.

In the proposed methodology, no relaxation terms are involved. In that case, boundary values do not relax towards any imposed mean target values. Even if no drift of boundary values was observed in the tests considered in the present work, the study of this topic has yet to be developed.

Acknowledgements. The financial support of the Conseil Régional d'Aquitaine is gratefully acknowledged. 


\section{Appendix A: Two time scales low Mach number asymptotics of wave amplitudes}

Reference pressure $p_{\mathrm{r}}$, density $\varrho_{\mathrm{r}}$ and velocity $v_{\mathrm{r}}$ thought of as a convective quantity, are introduced. A reference Mach number is then defined as $\mathrm{M}_{\mathrm{r}}=v_{\mathrm{r}} / \sqrt{p_{\mathrm{r}} / \varrho_{\mathrm{r}}}$. Reference length $l_{\mathrm{r}}$, thought of as convective quantity, is also considered, as well as reference duration defined as $t_{\mathrm{r}}=l_{\mathrm{r}} / v_{\mathrm{r}}$. The LODI equations (2) become in dimensionless form:

$$
\begin{aligned}
\partial_{\check{t}} \check{\varrho}+\mathrm{M}_{\mathrm{r}} \frac{\check{\varrho}}{2 \check{c}}\left(\check{\mathcal{L}}_{1}+\check{\mathcal{L}}_{3}\right)+\check{\mathcal{L}}_{2} & =0 \\
\partial_{\check{t}} \check{v}+\frac{1}{2}\left(\check{\mathcal{L}}_{3}-\check{\mathcal{L}}_{1}\right) & =0 \\
\partial_{\check{t}} \check{p}+\mathrm{M}_{\mathrm{r}} \frac{\check{\varrho} \check{c}}{2}\left(\check{\mathcal{L}}_{1}+\check{\mathcal{L}}_{3}\right) & =0
\end{aligned}
$$

where $`$ denotes dimensionless quantities. The dimensionless wave amplitudes read in temporal form:

$$
\begin{aligned}
\check{\mathcal{L}}_{1} & =-\left(\frac{1}{\mathrm{M}_{\mathrm{r}}} \frac{1}{\check{\varrho} \check{c}} \partial_{\check{t}} \check{p}-\partial_{\check{t}} \check{v}\right) \\
\check{\mathcal{L}}_{2} & =-\left(\partial_{\check{t}} \check{\varrho}-\frac{1}{\check{c}^{2}} \partial_{\check{t}} \check{p}\right) \\
\check{\mathcal{L}}_{3} & =-\left(\frac{1}{\mathrm{M}_{\mathrm{r}}} \frac{1}{\check{\varrho} \check{c}} \partial_{\check{t}} \check{p}+\partial_{\check{t}} \check{v}\right)
\end{aligned}
$$

A time variable relevant to capture rapid acoustic fluctuations at low Mach number $\mathrm{M}_{\mathrm{r}}$ is defined as

$$
\check{\tau}=\check{t} / \mathrm{M}_{\mathrm{r}}
$$

If $\mathrm{M}_{\mathrm{r}} \ll 1$, the pressure variable is assumed to be expanded as

$$
\check{p}\left(\check{x}, \check{t}, \mathrm{M}_{\mathrm{r}}\right)=\sum_{n=0}^{N} \mathrm{M}_{\mathrm{r}}{ }^{n} \check{p}^{(n)}(\check{x}, \check{t}, \check{\tau})+o\left(\mathrm{M}_{\mathrm{r}}{ }^{N}\right), \quad N=0,1,2
$$

with similar expansions for density and velocity. Substituting these expansions in expressions (A.1) and setting

$$
\begin{aligned}
& \check{c}^{(0)}=\sqrt{\frac{\gamma \check{p}^{(0)}}{\check{\varrho}^{(0)}}}, \quad \check{c}^{(1)}=\frac{1}{2} \check{c}^{(0)}\left(\frac{\check{p}^{(1)}}{\check{p}^{(0)}}-\frac{\check{\varrho}^{(1)}}{\check{\varrho}^{(0)}}\right), \\
& \check{c}^{(2)}=-\frac{1}{8} \check{c}^{(0)}\left(\frac{\check{p}^{(1)}}{\check{p}^{(0)}}-\frac{\check{\varrho}^{(1)}}{\check{\varrho}^{(0)}}\right)^{2}
\end{aligned}
$$

one obtains

$$
\check{\mathcal{L}}_{1}=\mathrm{M}_{\mathrm{r}}{ }^{-2} \check{\mathcal{L}}_{1}^{(-2)}+\mathrm{M}_{\mathrm{r}}{ }^{-1} \check{\mathcal{L}}_{1}^{(-1)}+\check{\mathcal{L}}_{1}^{(0)}+o(1)
$$

where

$$
\begin{aligned}
& \check{\mathcal{L}}_{1}^{(-2)}=-\frac{1}{(\varrho \check{c})^{(0)}} \partial_{\check{\tau}} \check{p}^{(0)} \\
& \check{\mathcal{L}}_{1}^{(-1)}=-\frac{1}{(\varrho \check{\varrho} \check{c})^{(0)}}\left(\partial_{\breve{t}} \check{p}^{(0)}+\partial_{\check{\tau}} \check{p}^{(1)}\right) \\
& +\frac{(\varrho \check{c} \check{c})^{(1)}}{(\varrho \check{c})^{(0)}} \partial_{\check{\tau}} \check{p}^{(0)}+\partial_{\check{\tau}} \check{v}^{(0)} \\
& \check{\mathcal{L}}_{1}^{(0)}=-\frac{1}{(\varrho \check{\varrho} \check{c})^{(0)}}\left(\partial_{\check{t}} \check{p}^{(1)}+\partial_{\check{\tau}} \check{p}^{(2)}\right) \\
& +\frac{(\varrho \check{c} \check{c})^{(1)}}{(\varrho \check{c})^{(0)}}\left(\partial_{\check{t}} \check{p}^{(0)}+\partial_{\check{\tau}} \check{p}^{(1)}\right) \\
& -\left(\left(\frac{(\check{\varrho} \check{c})^{(1)}}{(\check{\varrho} \check{c})^{(0)}}\right)^{2}-\frac{(\check{\varrho} \check{c})^{(2)}}{(\varrho \check{c})^{(0)}}\right) \partial_{\check{\tau}} \check{p}^{(0)} \\
& +\partial_{\check{t}} \check{v}^{(0)}+\partial_{\check{\tau}} \check{v}^{(1)}
\end{aligned}
$$

One also obtains

$$
\check{\mathcal{L}}_{2}=\mathrm{M}_{\mathrm{r}}{ }^{-1} \check{\mathcal{L}}_{2}^{(-1)}+\check{\mathcal{L}}_{2}^{(0)}+\mathrm{M}_{\mathrm{r}} \check{\mathcal{L}}_{2}^{(1)}+o\left(\mathrm{M}_{\mathrm{r}}\right)
$$

where

$$
\begin{aligned}
& \check{\mathcal{L}}_{2}^{(-1)}=-\left(\partial_{\check{\tau}} \check{\varrho}^{(0)}-\frac{1}{\left(\check{c}^{(0)}\right)^{2}} \partial_{\check{\tau}} \check{p}^{(0)}\right) \\
& \check{\mathcal{L}}_{2}^{(0)}=-\left(\partial_{\check{t}} \check{\varrho}^{(0)}+\partial_{\check{\tau}} \check{\varrho}^{(1)}-\frac{1}{\left(\check{c}^{(0)}\right)^{2}}\left(\partial_{\check{t}} \check{p}^{(0)}+\partial_{\check{\tau}} \check{p}^{(1)}\right.\right. \\
& \left.\left.-\frac{\left(\check{c}^{(1)}\right)^{2}}{\left(\check{c}^{(0)}\right)^{2}} \partial_{\check{\tau}} \check{p}^{(0)}\right)\right) \text {, }
\end{aligned}
$$

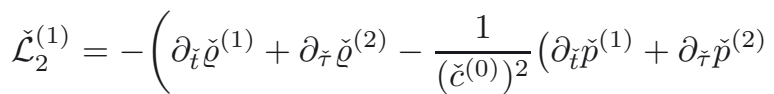

$$
\begin{aligned}
& \left.\left.-\frac{\left(\check{c}^{(1)}\right)^{2}}{\left(\check{c}^{(0)}\right)^{2}}\left(\partial_{\check{t}} \check{p}^{(0)}+\partial_{\check{\tau}} \check{p}^{(1)}\right)\right)\right)
\end{aligned}
$$

and

$$
\check{\mathcal{L}}_{3}=\mathrm{M}_{\mathrm{r}}{ }^{-2} \check{\mathcal{L}}_{3}^{(-2)}+\mathrm{M}_{\mathrm{r}}{ }^{-1} \check{\mathcal{L}}_{3}^{(-1)}+\check{\mathcal{L}}_{3}^{(0)}+o(1)
$$

where

$$
\begin{aligned}
& \check{\mathcal{L}}_{3}^{(-2)}=-\frac{1}{(\check{\varrho} \check{c})^{(0)}} \partial_{\check{\tau}} \check{p}^{(0)} \\
& \check{\mathcal{L}}_{3}^{(-1)}=-\frac{1}{(\varrho \check{c})^{(0)}}\left(\partial_{\check{t}} \check{p}^{(0)}+\partial_{\check{\tau}} \check{p}^{(1)}\right) \\
& +\frac{(\varrho \check{\varrho} \check{c})^{(1)}}{(\varrho \check{c} \check{c})^{(0)}} \partial_{\check{\tau}} \check{p}^{(0)}-\partial_{\check{\tau}} \check{v}^{(0)} \\
& \check{\mathcal{L}}_{3}^{(0)}=-\frac{1}{(\varrho \check{c})^{(0)}}\left(\partial_{\check{t}} \check{p}^{(1)}+\partial_{\check{\tau}} \check{p}^{(2)}\right) \\
& +\frac{(\varrho \check{\varrho} \check{c})^{(1)}}{(\varrho \check{\varrho})^{(0)}}\left(\partial_{\check{t}} \check{p}^{(0)}+\partial_{\check{\tau}} \check{p}^{(1)}\right) \\
& -\left(\left(\frac{(\varrho \check{\varrho} \check{c})^{(1)}}{(\varrho \check{\varrho})^{(0)}}\right)^{2}-\frac{(\varrho \check{\varrho} \check{c})^{(2)}}{(\varrho \check{\varrho})^{(0)}}\right) \partial_{\check{\tau}} \check{p}^{(0)} \\
& -\left(\partial_{\check{t}} \check{v}^{(0)}+\partial_{\check{\tau}} \check{v}^{(1)}\right)
\end{aligned}
$$


On the other hand, the dimensionless spatial form of the wave amplitudes reads

$$
\begin{aligned}
& \check{\mathcal{L}}_{1}=\left(\check{v}-\frac{\check{c}}{\mathrm{M}_{\mathrm{r}}}\right)\left(\frac{1}{\mathrm{M}_{\mathrm{r}}} \frac{1}{\check{\varrho} \check{c}} \partial_{\check{x}} \check{p}-\partial_{\check{x}} \check{v}\right) \\
& \check{\mathcal{L}}_{2}=\check{v}\left(\partial_{\check{x}} \check{\varrho}-\frac{1}{\check{c}^{2}} \partial_{\check{x}} \check{p}\right) \\
& \check{\mathcal{L}}_{3}=\left(\check{v}+\frac{\check{c}}{\mathrm{M}_{\mathrm{r}}}\right)\left(\frac{1}{\mathrm{M}_{\mathrm{r}}} \frac{1}{\check{\varrho} \check{c}} \partial_{\check{x}} \check{p}+\partial_{\check{x}} \check{v}\right)
\end{aligned}
$$

Using as before the two time scales low Mach number expansions, one obtains

$$
\begin{aligned}
& \check{\mathcal{L}}_{1}^{(-2)}=-\frac{1}{\check{\varrho}^{(0)}} \partial_{\check{x}} \check{p}^{(0)} \\
& \check{\mathcal{L}}_{1}^{(-1)}=\left(\check{v}^{(0)}-\check{c}^{(1)}\right) \frac{1}{(\check{\varrho} \check{c})^{(0)}} \partial_{\check{x}} \check{p}^{(0)} \\
& -\check{c}^{(0)}\left(\frac{1}{(\varrho \check{c} \check{c})^{(0)}} \partial_{\check{x}} \check{p}^{(1)}\right. \\
& \left.-\frac{1}{(\varrho \check{\varrho})^{(0)}} \frac{(\check{\varrho} \check{c})^{(1)}}{(\check{\varrho} \check{c})^{(0)}} \partial_{\check{x}} \check{p}^{(0)}-\partial_{\check{x}} \check{v}^{(0)}\right) \\
& \check{\mathcal{L}}_{1}^{(0)}=-\check{c}^{(0)}\left(\frac{1}{(\varrho \check{\varrho})^{(0)}} \partial_{\check{x}} \check{p}^{(2)}-\frac{1}{(\varrho \check{c})^{(0)}} \frac{(\varrho \check{\varrho})^{(1)}}{(\varrho \check{c})^{(0)}} \partial_{\check{x}} \check{p}^{(1)}\right. \\
& \left.-\frac{1}{(\varrho \check{\varrho})^{(0)}} \frac{(\check{\varrho} \check{c})^{(2)}}{(\varrho \check{c})^{(0)}} \partial_{\check{x}} \check{p}^{(0)}-\partial_{\check{x}} \check{v}^{(1)}\right) \\
& +\left(\check{v}^{(0)}-\check{c}^{(1)}\right)\left(\frac{1}{(\varrho \varrho \check{c})^{(0)}} \partial_{\check{x}} \check{p}^{(1)}\right. \\
& \left.-\frac{1}{(\varrho \check{\varrho})^{(0)}} \frac{(\check{\varrho} \check{c})^{(1)}}{(\check{\varrho} \check{c})^{(0)}} \partial_{\check{x}} \check{p}^{(0)}-\partial_{\check{x}} \check{v}^{(0)}\right) \\
& +\left(\check{v}^{(1)}-\check{c}^{(2)}\right) \frac{1}{(\check{\varrho} \check{c})^{(0)}} \partial_{\breve{x}} \check{p}^{(0)}
\end{aligned}
$$

$$
\begin{aligned}
\check{\mathcal{L}}_{2}^{(-1)}= & 0, \\
\check{\mathcal{L}}_{2}^{(0)} & =\check{v}^{(0)}\left(\partial_{\check{x}} \check{\varrho}^{(0)}-\frac{1}{\left(\check{c}^{(0)}\right)^{2}} \partial_{\check{x}} \check{p}^{(0)}\right), \\
\check{\mathcal{L}}_{2}^{(1)}= & \check{v}^{(0)}\left(\partial_{\check{x}} \check{\varrho}^{(1)}-\frac{1}{\left(\check{c}^{(0)}\right)^{2}}\left(\partial_{\check{x}} \check{p}^{(1)}-\frac{\left(\check{c}^{(1)}\right)^{2}}{\left(\check{c}^{(0)}\right)^{2}} \partial_{\check{x}} \check{p}^{(0)}\right)\right) \\
& +\check{v}^{(1)}\left(\partial_{\check{x}} \check{\varrho}^{(0)}-\frac{1}{\left(\check{c}^{(0)}\right)^{2}} \partial_{\check{x}} \check{p}^{(0)}\right)
\end{aligned}
$$

$$
\begin{aligned}
\check{\mathcal{L}}_{3}^{(-2)}= & \frac{1}{\check{\varrho}^{(0)}} \partial_{\check{x}} \check{p}^{(0)} \\
\check{\mathcal{L}}_{3}^{(-1)}= & \left(\check{v}^{(0)}+\check{c}^{(1)}\right) \frac{1}{(\check{\varrho} \check{c})^{(0)}} \partial_{\check{x}} \check{p}^{(0)} \\
& +\check{c}^{(0)}\left(\frac{1}{(\check{\varrho} \check{c})^{(0)}} \partial_{\check{x}} \check{p}^{(1)}\right. \\
& \left.-\frac{1}{(\varrho \check{c} \check{c})^{(0)}} \frac{(\check{\varrho} \check{c})^{(1)}}{(\varrho \check{c})^{(0)}} \partial_{\check{x}} \check{p}^{(0)}+\partial_{\check{x}} \check{v}^{(0)}\right)
\end{aligned}
$$

$$
\begin{aligned}
& \check{\mathcal{L}}_{3}^{(0)}=\check{c}^{(0)}\left(\frac{1}{(\check{\varrho} \check{c})^{(0)}} \partial_{\check{x}} \check{p}^{(2)}-\frac{1}{(\check{\varrho} \check{c})^{(0)}} \frac{(\varrho \check{\varrho} \check{c})^{(1)}}{(\check{\varrho} \check{c})^{(0)}} \partial_{\check{x}} \check{p}^{(1)}\right. \\
& \left.-\frac{1}{(\check{\varrho} \check{c})^{(0)}} \frac{(\varrho \check{\check{c}})^{(2)}}{(\varrho \check{c})^{(0)}} \partial_{\check{x}} \check{p}^{(0)}+\partial_{\check{x}} \check{v}^{(1)}\right) \\
& +\left(\check{v}^{(0)}+\check{c}^{(1)}\right)\left(\frac{1}{(\varrho \check{c})^{(0)}} \partial_{\check{x}} \check{p}^{(1)}\right. \\
& \left.-\frac{1}{(\check{\varrho} \check{c})^{(0)}} \frac{(\check{\varrho} \check{c})^{(1)}}{(\check{\varrho} \check{c})^{(0)}} \partial_{\check{x}} \check{p}^{(0)}+\partial_{\check{x}} \check{v}^{(0)}\right) \\
& +\left(\check{v}^{(1)}+\check{c}^{(2)}\right) \frac{1}{(\varrho \check{\varrho})^{(0)}} \partial_{\check{x}} \check{p}^{(0)}
\end{aligned}
$$

With equations (A.1), by matching the previous expansions in temporal and spatial forms one then obtains:

$$
\begin{aligned}
& \partial_{\check{\tau}} \check{\varrho}^{(0)}=0
\end{aligned}
$$



$$
\begin{aligned}
& \partial_{\check{x}} \check{p}^{(0)}=0 \\
& \partial_{\check{\tau}} \check{p}^{(0)}=0 \\
& \partial_{\check{\tau}} \check{v}^{(0)}+\frac{1}{\check{\varrho}^{(0)}} \partial_{\check{x}} \check{p}^{(1)}=0 \\
& \mathrm{~d}_{\check{t}} \check{p}^{(0)}+\partial_{\check{\tau}} \check{p}^{(1)}+\gamma p^{(0)} \partial_{\check{x}} \check{v}^{(0)}=0
\end{aligned}
$$

The different pressure components can now be identified. Observe first that $\check{p}^{(0)}$ depends on $\check{t}$, which denotes the time at the convective scale, but not on $\check{x}$ and $\check{\tau}$, which stands for the acoustic scale. From equation (A.16a), the zeroth-order density does not depend on $\check{\tau}$. Then, from equations (A.16d)-(A.16f),

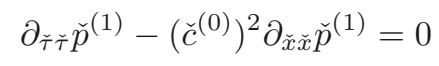

where $\left(\check{c}^{(0)}\right)^{2}=\left(\gamma p^{(0)} / \varrho^{(0)}\right)(x, t)$. As solution of the linear acoustic waves equation (A.17) with non-constant propagation speed $c^{(0)}$, the first-order pressure $p^{(1)}$ is identified as the acoustic pressure. Assuming that the integral of $\partial_{\check{\tau}} \check{p}^{(1)}$ over the computational domain is negligible, $\check{p}^{(0)}=\check{p}^{(0)}(t)$ can then be calculated with the integral of $v^{(0)}$ on the boundary of the computational domain. The zeroth-order pressure $\check{p}^{(0)}$ is thus identified as the thermodynamic pressure. Finally, the second-order pressure $\check{p}^{(2)}$ is identified as the hydrodynamic pressure.

The frozen turbulent inflow hypothesis is written as transport equations for density and velocity at the zerothorder and at the convective time scale,

$$
\begin{aligned}
& \partial_{\check{t}} \check{\varrho}^{(0)}+\check{V} \partial_{\check{x}} \check{\varrho}^{(0)}=0 \\
& \partial_{\check{t}} \check{v}^{(0)}+\check{V} \partial_{\check{x}} \check{v}^{(0)}=0
\end{aligned}
$$

In equations (A.18), we can take for example $\check{V}=\check{v}^{(0)}$, but this choice is not mandatory for our analysis. The point is that the following result of the analysis by Prosser [8] under hypothesis (A.18b) (see Eq. (32e), p. 751 in [8]):

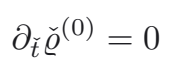


is equivalent, through equation (A.18a), to

$$
\partial_{\check{x}} \check{\varrho}^{(0)}=0
$$

With equations (A.14a), (A.16c) and (A.20),

$$
\begin{aligned}
& \check{\mathcal{L}}_{2}^{(-1)}=0, \\
& \check{\mathcal{L}}_{2}^{(0)}=0, \\
& \check{\mathcal{L}}_{2}^{(1)}=\check{v}^{(0)}\left(\partial_{\check{x}} \check{\varrho}^{(1)}-\frac{1}{\left(\check{c}^{(0)}\right)^{2}} \partial_{\check{x}} \check{p}^{(1)}\right)
\end{aligned}
$$

Suppose that $v^{\dagger}$ is expanded as in equation (A.4). Concerning the NR-NSCBC method (see Eqs. (6)), few calculations lead to:

$$
\begin{aligned}
\check{\mathcal{L}}_{2}^{(-1)}= & -(\gamma-1) \frac{\check{\varrho}^{(0)}}{\check{c}^{(0)}} \partial_{\check{\tau}}\left(\check{v}^{\dagger}\right)^{(0)} \\
\check{\mathcal{L}}_{2}^{(0)}= & -(\gamma-1)\left\{\frac { \check { \varrho } ^ { ( 0 ) } } { \check { c } ^ { ( 0 ) } } \left[\partial_{\check{t}}\left(\check{v}^{\dagger}\right)^{(0)}+\partial_{\check{\tau}}\left(\check{v}^{\dagger}\right)^{(1)}\right.\right. \\
& \left.\left.-\frac{\check{c}^{(1)}}{\check{c}^{(0)}} \partial_{\check{\tau}}\left(\check{v}^{\dagger}\right)^{(0)}\right]+\frac{\check{\varrho}^{(1)}}{\check{c}^{(0)}} \partial_{\check{\tau}}\left(\check{v}^{\dagger}\right)^{(0)}\right\}
\end{aligned}
$$

which do not match the low Mach number asymptotics under the frozen turbulent inflow hypothesis that led to equations (A.21a) and (A.21b).

Returning to $\check{\mathcal{L}}_{3}$, from equations (A.11) and (A.16),

$$
\begin{aligned}
& \check{\mathcal{L}}_{3}^{(-2)}=0 \\
& \check{\mathcal{L}}_{3}^{(-1)}=-\partial_{\check{\tau}} \check{v}^{(0)}-\frac{1}{(\varrho \check{c})^{(0)}}\left(\partial_{\breve{t}} \check{p}^{(0)}+\partial_{\check{\tau}} \check{p}^{(1)}\right) \\
& \check{\mathcal{L}}_{3}^{(0)}=-\frac{1}{(\check{\varrho} \check{c})^{(0)}}\left(\partial_{\check{t}} \check{p}^{(1)}+\partial_{\check{\tau}} \check{p}^{(2)}\right) \\
& +\frac{(\varrho \check{\varrho} \check{c})^{(1)}}{(\varrho \check{\varrho})^{(0)}}\left(\partial_{\check{t}} \check{p}^{(0)}+\partial_{\check{\tau}} \check{p}^{(1)}\right)-\left(\partial_{\check{t}} \check{v}^{(0)}+\partial_{\check{\tau}} \check{v}^{(1)}\right)
\end{aligned}
$$

Suppose again that $v^{\dagger}$ is expanded as in equation (A.4). Then, from equations (4), (A.7b), (A.7c) and (A.16d),

$$
\begin{aligned}
& \partial_{\check{\tau}} \check{v}^{(0)}=2 \partial_{\check{\tau}}\left(\check{v}^{\dagger}\right)^{(0)}-\frac{1}{(\check{\varrho} \check{c})^{(0)}}\left(\mathrm{d}_{\check{t}} \check{p}^{(0)}+\partial_{\check{\tau}} \check{p}^{(1)}\right), \\
& \partial_{\breve{t}} \check{v}^{(0)}+\partial_{\check{\tau}} \check{v}^{(1)}=2 \partial_{\check{t}}\left(\check{v}^{\dagger}\right)^{(0)}+2 \partial_{\check{\tau}}\left(\check{v}^{\dagger}\right)^{(1)}-\frac{1}{(\varrho \varrho \check{c})^{(0)}}\left(\partial_{\breve{t}} \check{p}^{(1)}\right.
\end{aligned}
$$

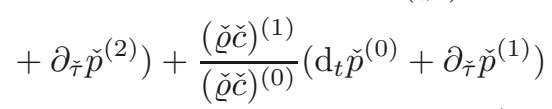

and with equations (A.23), one obtains:

$$
\begin{aligned}
\check{\mathcal{L}}_{3}^{(-2)} & =0 \\
\check{\mathcal{L}}_{3}^{(-1)} & =-2 \partial_{\check{\tau}}\left(\check{v}^{\dagger}\right)^{(0)} \\
\check{\mathcal{L}}_{3}^{(0)} & =-2 \partial_{\check{t}}\left(\check{v}^{\dagger}\right)^{(0)}-2 \partial_{\check{\tau}\left(\check{v}^{\dagger}\right)^{(1)}}
\end{aligned}
$$

\section{References}

[1] K.W. Thompson, Time dependent boundary conditions for hyperbolic systems, J. Comput. Phys. 68 (1987) 1-24

[2] T.J. Poinsot, S.K. Lele, Boundary conditions for direct simulations of compressible viscous flow, J. Comput. Phys. 101 (1992) 104-129

[3] D.H. Rudy, J.C. Strikwerda, A nonreflecting outflow boundary condition for subsonic Navier-Stokes calculations, J. Comput. Phys. 36 (1980) 55-70

[4] L. Selle, F. Nicoud, T. Poinsot, Actual impedance of nonreflecting boundary conditions: Implications for computation of resonators, AIAA J. 42 (2004) 958-964

[5] Y. Moguen, P. Bruel, E. Dick, Semi-implicit characteristic-based boundary treatment for acoustics in low Mach number flows, J. Comput. Phys. 255 (2013) 339-361

[6] S. Pirozzoli, T. Colonius, Generalized characteristic relaxation boundary conditions for unsteady compressible flow simulations, J. Comput. Phys. 248 (2013) 109-126

[7] W. Polifke, C. Wall, P. Moin, Partially reflecting and non-reflecting boundary conditions for simulation of compressible viscous flow, J. Comput. Phys. 213 (2006) 437-449

[8] R. Prosser, Towards improved boundary conditions for the DNS and LES of turbulent subsonic flows, J. Comput. Phys. 222 (2005) 469-474

[9] N. Guézennec, T. Poinsot, Acoustically nonreflecting and reflecting boundary conditions for vorticity injection in compressible solvers, AIAA J. 47 (2009) 1709-1722

[10] G.I. Taylor, The spectrum of turbulence, Proc. R. Soc. Lond. A 164 (1938) 476-490

[11] Y. Moguen, T. Kousksou, P. Bruel, J. Vierendeels, E. Dick, Pressure-velocity coupling allowing acoustic calculation in low Mach number flow, J. Comput. Phys. 231 (2012) 5522-5541

[12] W.Z. Shen, J.A. Michelsen, J.N. Sørensen, Improved Rhie-Chow interpolation for unsteady flow computations, AIAA J. 39 (2001) 2406-2409

[13] L. Biferale, G. Boffetta, A. Celani, A. Crisanti, A. Vulpiani, Mimicking a turbulent signal: Sequential multiaffine processes, Phys. Rev. E 57 (1998) R6261-R6264

[14] J.D. Wilson, Y. Zhuang, Restriction on the timestep to be used in stochastic Lagrangian models of turbulent dispersion, Bound.-Lay. Meteorol. 49 (1989) 309-316 